\title{
Utility of the Korean Modified Early Warning Score (KMEWS) as a Triage Screening Tool in the Emergency Department in Patients with Infection
}

\section{Se kwang Oh (D13744@hanmail.net )}

Department of Emergency Medicine, Chungnam National University Sejong Hospital, Sejong, Republic of korea

\section{Seung Ryu}

Department of Emergency Medicine, Chungnam National University Hospital, Daejeon, Republic of korea

\section{So young Jeon}

Department of Emergency Medicine, Chungnam National University Hospital, Daejeon, Republic of korea

\section{Bong Kyu Lee}

Department of Emergency Medicine, Chungnam National University Hospital, Daejeon, Republic of korea

\section{Research Article}

Keywords: Emergency department, Triage, Septic shock, Prediction, Mortality, Intensive care, Korean Modified Early Warning Score

Posted Date: May 27th, 2021

DOl: https://doi.org/10.21203/rs.3.rs-538791/v1

License: (9) This work is licensed under a Creative Commons Attribution 4.0 International License. Read Full License 


\section{Abstract}

Background: In patients with sepsis, early resuscitation and intensive care are associated with a lower mortality rate compared with late resuscitation. We evaluated the utility of the Korean Modified Early Warning Score (KMEWS), which combines the Modified Early Warning Score (MEWS) and the Korean Triage and Acuity Scale (KTAS), as a triage tool to screen for infection in patients visiting the emergency department (ED).

Methods: We retrospectively reviewed data extracted from electronic medical records. Patients aged $\geq 18$ years, with infection, who were admitted to hospital via the ED between January 1, 2018, and December 31, 2019 were eligible for inclusion. The MEWS, quick Sepsis-related Organ Failure Assessment (qSOFA), and Mortality in Emergency Department Sepsis (MEDS) of each patient were calculated. The KTAS score was 5, 4, 3, 2, and 1 point, for Levels 1, 2, 3, 4, and 5, respectively. The KMEWS was calculate as the sum of the KTAS score and the MEWS. In addition, the participants were assigned to a septic shock group and a non-septic group for intergroup comparisons.

Results: The area under the curve (AUC) values for predicting septic shock were: KMEWS, 0.910 (95\% confidence interval [Cl], 0.902-0.918); MEWS, 0.896 (95\% Cl, 0.887-0.904); KTAS score, $0.809(95 \% \mathrm{Cl}$, $0.798-0.819)$; and the MEDS, 0.927 (95\% $\mathrm{Cl}, 0.919-0.934)$. The AUC values for predicting mortality were: KMEWS, 0.752 (95\% Cl, 0.740-0.764); MEWS, 0.717 (95\% Cl, 0.704-0.729); KTAS score, 0.764 (95\% Cl, 0.752-0.776); and the MEDS, $0.844(95 \% \mathrm{Cl}, 0.834-0.854)$. The AUC values for predicting intensive care unit admission were: KMEWS, 0.826 (95\% Cl, 0.816-0.837); MEWS, 0.782 (95\% Cl, 0.770-0.793); KTAS score, 0.821 ( $95 \% \mathrm{Cl}, 0.810-0.831)$; and the MEDS, 0.839 (95\% Cl, 0.829-0.849).

Conclusion: The KMEWS is a useful triage screening tool for determining clinical severity in ED patients with infection.

\section{Background}

Sepsis is an inflammatory disease caused by a reaction of the immune system, which can be lifethreatening and responsible for $20 \%$ of all hospital deaths each year [1, 2]. In patients with sepsis, early resuscitation and intensive care are associated with a lower mortality rate compared with late resuscitation [3]. Therefore, the recognition of sepsis and timely initiation of evidence-based protocols are critical $[4,5]$. However, diagnosis of sepsis using the Sepsis-3 definitions is a time-consuming in overcrowded emergency departments (EDs) [6]. Several markers for predicting septic shock are known, but in most of them require laboratory tests that take time. Therefore, a tool is required that can predict septic shock early in the triage stage [7-11].

The Modified Early Warning Score (MEWS) is a simple physiological score used to screen patients at risk of clinical deterioration using values of body temperature, blood pressure, pulse rate, respiratory rate, and level of consciousness, and is used as a screening tool for septic shock $[12,13]$ (Supplementary Table 1). In Korea, the Korean Triage and Acuity Scale (KTAS) based on the Canadian Triage and Acuity Scales 
(CTAS) has been developed and used since 2016. The KTAS is a combination of variables, including vital signs and chief complaints, and serves as a tool for determining the severity and priority of treatment in the ED $[14,15]$ (Supplementary Table 2).

Therefore, this study was conducted to investigate whether the KMEWS, which combines the MEWS and the KTAS, is useful as a triage tool to screen patients with infection in the ED.

\section{Materials And Methods}

\section{Study design and setting}

We retrospectively reviewed the data extracted from electronic medical records. The study sample included patients aged $\geq 18$ years with infection who were admitted to hospital via the ED between January 1, 2018, and December 31, 2019, at a tertiary-care university hospital with 1350 beds in Daejeon, Korea. The ED provides medical care to approximately 55,000 patients per year. Patients with missing data were excluded.

The diagnosis of infection was confirmed using the relevant International Classification of Diseases, 10th Revision (ICD-10) codes in the medical records. Patients with any of the following infection-related ICD-10 codes were eligible for enrolment: A00-B99, G00-09, I00-02, I30-33, I38-41, J00-J22, J36, J37, J40J43, J68, J69, J80, J85-J86, K11-12, K35-37, K57, K61, K63, K65, K67, K75, K77.0, K80-81, K83.0, K85, L00-08, M00-03, M86, N10, N12, N13.6, N16.0, N28.84-28.86, N30, N34, N39.0, N41, N45, N61, N70-74, and 091.

Sepsis patients were defined as the presence of two or three of the three quick Sepsis-related Organ Failure Assessment (qSOFA) clinical criteria (altered mentation, respiratory rate $\geq 22$ breaths $/ \mathrm{min}$, and systolic blood pressure $\leq 100 \mathrm{mmHg}$ ) (16). Among patients with sepsis, septic shock was clinically defined as a case where vasopressor is required to maintain a mean arterial pressure of $\geq 65 \mathrm{mmHg}$, and serum lactate level $>2 \mathrm{mmol} / \mathrm{L}(>18 \mathrm{mg} / \mathrm{dL})$ [16].

Only clinical data were extracted, and no personal or identifiable information was recorded. The need for informed consent was waived by the Institutional Review Board consent guideline of Chungnam National University Hospital because of the retrospective study design and the use of anonymized data. The study was approved by the Chungnam National University Hospital Institutional Review Board (approval no. : 2020-10-059), and all methods were performed in accordance with the relevant guidelines and regulations. Due to the retrospective nature of the study, the Institutional Review Board of Chungnam National University Hospital waived the need for written informed consent.

\section{Data collection and outcome measures}

We collected clinical data from the electronic medical records. The information included age, sex, systolic arterial pressure $(\mathrm{mmHg})$, respiratory rate (breaths $/ \mathrm{min})$, body temperature $\left({ }^{\circ} \mathrm{C}\right)$, and mental status. We calculated the Charlson Comorbidity Index $(\mathrm{CCl})$, which categorizes the comorbidities of patients based 
on the International Classification of Diseases diagnosis codes found in the administrative data [17], for each patient. In addition, we calculated the Mortality in Emergency Department Sepsis (MEDS) score, the MEWS, the KTAS score, and the qSOFA score of each patient $[7-9,12,13]$. The number of points on the KTAS score was 5, 4, 3, 2, or 1 for Levels 1, 2, 3, 4, and 5, respectively. The KMEWS was calculated as the sum of the KTAS score and the MEWS. In addition, we divided the participants into septic shock and nonseptic groups, and conducted intergroup comparisons.

\section{Data analysis}

Continuous variables were analyzed using the Student's t-test or the Mann-Whitney Utest, and categorical variables were analyzed using chi-square or Fisher's exact tests. Multivariable logistic regression was performed to identify predictive factors for septic shock, using variables that had previously been reported to be significantly associated with septic shock.

We generated receiver-operating characteristic (ROC) curves, and determined the area under the curve (AUC) for individual measures (the KMEWS, KTAS, MEWS, and the MEDS) associated with septic shock, intensive care unit (ICU) admission, and mortality. All statistical analyses were performed using SPSS software, Version 19.0 (IBM Corp., Armonk, NY, USA) and MedCalc 14.8.1 (MedCalc software, BVBA, Ostend, Belgium). $P$ values $<0.05$ were considered statistically significant.

\section{Results}

\section{Patient characteristics}

A total of 19,214 patients were enrolled during the study period, of whom 5,093 were included in the analytic dataset (Fig. 1). Among them, 395 and 4,698 patients were in the septic shock and non-septic shock groups, respectively.

\section{Comparison of characteristics of the septic shock group and non-septic shock group}

Intergroup comparisons of the septic shock group and non-septic shock group showed no significant difference in sex; however, the participants in the septic shock group were older (Median [IQR], 78 [68-83] years vs 61 [42.0-76.0] years, $p<0.001)$. In the septic shock group, systolic arterial pressure was lower than that in the non-septic shock group (Median [IQR], 99 [85-127] mmHg vs 125 [111-140] mmHg, $p<0.001)$ was lower, and a higher proportion of patients had deteriorating mental status $(72.4 \% \mathrm{vs} 6.0 \%$, $p<0.001)$. The septic shock group had a higher CCI (Median [IQR], 5 [ $4-6]$ vs 2 [0-5], $p<0.001)$. The laboratory results were generally poor in the septic shock group (Table 1). The septic shock group had a significantly higher proportion of patients with KTAS Level 1 or $2(7.3 \%$ vs $56.1 \%, p<0.001)$ and a higher MEWS, KTAS score, and KMEWS (Table 1).

\section{Variables associated with septic shock using multivariable logistic regression and receiver-operating characteristic curve analysis}


Multivariable logistic regression revealed that age, transfer from a long-term care facility (LTCF), KTAS score, and MEWS were significantly associated with septic shock, but sex and CCI were not (Table 2).

In the ROC analysis, the AUC values of factors associated with septic shock were: KMEWS, $0.910(95 \% \mathrm{Cl}$, 0.902-0.918); MEWS, 0.896 (95\% Cl, 0.887-0.904); KTAS score, 0.809 ( $95 \% \mathrm{Cl}, 0.798-0.819)$; and the MEDS score, 0.927 (95\% Cl, 0.919-0.934) (Fig. 2). The optimal cut-off value of KMEWS for predicting septic shock was 7 (sensitivity, $81.3 \%$; specificity, $82.6 \%$ ). The respective AUC values of factors associated with mortality were KMEWS, 0.752 (95\% Cl, 0.740-0.764); MEWS, 0.717 (95\% Cl, 0.7040.729), KTAS score, 0.764 (95\% Cl, 0.752-0.776); and the MEDS, 0.844 (95\% Cl, 0.834-0.854) (Fig. 3). The respective AUC values of factors associated with ICU admission were: KMEWS, $0.826(95 \% \mathrm{Cl}$, 0.816-0.837); MEWS, 0.782 (95\% Cl, $0.770-0.793)$; KTAS score, 0.821 ( $95 \% \mathrm{Cl}, 0.810-0.831)$; and the MEDS, 0.839 (95\% Cl, 0.829-0.849) (Fig. 4).

\section{Discussion}

In the ED, triage helps predict the severity and determines the priority of patient treatment [18]. The wellknown triage scales are the Emergency Severity Index in the USA, the CTAS in Canada, the Australian Triage Scale in Australia, and the Manchester Triage Scale in the UK $[18,19]$. In Korea, the KTAS was developed based on the CTAS and implemented in 2015. The KTAS consists of a five-level system that classifies patients using a combination of variables, including vital signs and chief complaints $[14,15]$. Since the implementation of the KTAS, admission and disposition patterns have changed and reduced mortality in the ED [14].

Patients visit the ED with a wide variety of complaints, but the proportion of patients with infection is substantial $[20,21]$. As early diagnosis of sepsis or septic shock is one of the most important factors affecting the success of treatment, many scoring systems using various markers have been used [22, 23]. However, the current triage tools are inadequate for determining the severity and prognosis of patients presenting to the ED with infection $[24,25]$.

The qSOFA score is an established screening tool for sepsis [6]. However, qSOFA is not suitable as a screening tool because of its low sensitivity [26]. The MEDS score consists of nine factors associated with a greater mortality risk, including age $>65$ years, altered mental status, and terminal illness. The MEDS score has moderate accuracy in predicting mortality in ED patients with suspected infection, and the MEDS is superior to MEWS in predicting mortality in this patient population [7-9]. However, as the nine factors making up the MEDS score include platelet count and neutrophil count, it takes time to obtain the score and it is thus not suitable for use as a triage screening tool in ED.

The Early Warning Score is a simple physiological scoring system that can be easily applied at the bedside [12]. The MEWS is used as a screening tool for septic shock patients who are at risk of clinical deterioration using values of temperature, blood pressure, pulse, respiratory rate, and level of consciousness. MEWS may be useful for screening patients with septic shock $[12,13,16,27]$. Moreover, the MEWS does not require laboratory test results; therefore, it is immediately available at triage. 
However, as the MEWS is somewhat non-specific and does not contain factors related to the chief complaint, there is a limitation in patients with infection. On the other hand, the KTAS includes the chief complaint and the vital signs, but the hemodynamic criteria are not subdivided. However, the KTAS and MEWS can be applied to ED triage because laboratories are not required. Therefore, we hypothesized that supplementing the physiological data with MEWS to KTAS could help determine prompt prognosis in infected patients.

As the KTAS includes both vital signs and chief complaints, and the MEWS includes vital signs, the KMEWS, the sum of the KTAS and the MEWS, has a weighting value for the initial vital signs. In the multivariable logistic regression analysis of this study, KTAS and KMEWS were independently associated with septic shock and showed similar odds ratios for septic shock. Therefore, the KMEWS was calculated by combining the two scores. As a result, KMEWS showed similar or higher AUC values for septic shock, ICU admission, and mortality compared to either the KTAS score or the MEWS alone. The MEDS had a slightly higher AUC value than the KMEWS, but it is unsuitable for use as a septic screening tool in the ED. Therefore, KMEWS could be a useful prognostic tool for triaging patients with suspected infection in the ED.

Nevertheless, this study had some limitations. First, it was a single-center observational study that included only patients admitted to the hospital via the ED. Patients who had transferred to another hospital or died in the ED were excluded. Therefore, the generalizability of our results may be limited. Second, it included a collection of retrospective data that could introduce potential information biases and had much missing data. Third, the sepsis diagnosis process was excluded because it was a retrospective study of patients already diagnosed with an infectious disease. Inclusion criteria in our study were based on ICD-10 codes related to infection, and no blood culture reports were available. The diagnosis of septic shock was defined as sepsis with a serum lactate level $>2 \mathrm{mmol} / \mathrm{L}$, which did not reflect the patient's volume state. Finally, there could have been inter-clinician variability in calculating the KTAS score and the MEWS during triage.

\section{Conclusion}

The KMEWS could be a useful triage screening tool and an early warning sign to predict poor prognosis in patients with infection. Prospective multicenter studies are necessary to validate these findings.

\section{Declarations}

\section{Acknowledgments}

Not applicable

\section{Authors' contributions}


Seung Ryu: Conceptualization, Formal analysis, Writing - original draft, Writing - review \& editing, Supervision. Se kwang Oh: Conceptualization, Writing - original draft, Supervision. Bong Kyu Lee: Data curation, Formal analysis. So young Jeon: Data curation, Formal analysis, Supervision. All authors approved the final version of the manuscript.

\section{Funding}

Not applicable.

\section{Availability of data and materials}

The datasets used or analyzed during the current study are not expected to be made available due to IRB approval restrictions but are available from the corresponding author on reasonable request.

\section{Ethics approval and consent to participate}

The study had the approval from the Chungnam National University Hospital Institutional Review Board (approval no.: 2020-10-059). Due to the retrospective nature of the study, the Institutional Review Board of Chungnam National University Hospital waived the need for written informed consent.

\section{Consent for publication}

Not applicable.

\section{Competing interests}

The authors declare that they have no competing interests.

\section{Author details}

${ }^{1}$ Department of Emergency Medicine, Chungnam National University Hospital, 282, Munhwa-ro, Jung-gu, Daejeon, Republic of korea . ${ }^{2}$ Department of Emergency Medicine, Chungnam National University Sejong Hospital, 20 Bodeum-7-ro, Sejong, Republic of korea.

\section{References}

1. Angus DC, Linde-Zwirble WT, Lidicker J, Clermont G, Carcillo J, Pinsky MR. Epidemiology of severe sepsis in the United States: analysis of incidence, outcome, and associated costs of care. Crit Care Med. 2001;29(7):1303-10.

2. Martin GS, Mannino DM, Moss M. The effect of age on the development and outcome of adult sepsis. Crit Care Med. 2006;34(1):15-21.

3. Jones AE, Brown MD, Trzeciak S, Shapiro NI, Garrett JS, Heffner AC, et al. The effect of a quantitative resuscitation strategy on mortality in patients with sepsis: a meta-analysis. Crit Care Med. 2008;36(10):2734-9. 
4. Gauer RL. Early recognition and management of sepsis in adults: the first six hours. Am Fam Physician. 2013;88(1):44-53.

5. Ferrer R, Artigas A, Suarez D, Palencia E, Levy MM, Arenzana A, et al. Effectiveness of treatments for severe sepsis: a prospective, multicenter, observational study. Am J Respir Crit Care Med. 2009;180(9):861-6.

6. Seymour CW, Liu VX, Iwashyna TJ, Brunkhorst FM, Rea TD, Scherag A, et al. Assessment of Clinical Criteria for Sepsis: For the Third International Consensus Definitions for Sepsis and Septic Shock (Sepsis-3). JAMA. 2016;315(8):762-74.

7. Shapiro NI, Wolfe RE, Moore RB, Smith E, Burdick E, Bates DW. Mortality in Emergency Department Sepsis (MEDS) score: a prospectively derived and validated clinical prediction rule. Crit Care Med. 2003;31(3):670-5.

8. Chang SH, Hsieh CH, Weng YM, Hsieh MS, Goh ZNL, Chen HY, et al. Performance Assessment of the Mortality in Emergency Department Sepsis Score, Modified Early Warning Score, Rapid Emergency Medicine Score, and Rapid Acute Physiology Score in Predicting Survival Outcomes of Adult Renal Abscess Patients in the Emergency Department. Biomed Res Int. 2018;2018:6983568.

9. Zhang G, Zhang K, Zheng X, Cui W, Hong Y, Zhang Z. Performance of the MEDS score in predicting mortality among emergency department patients with a suspected infection: a meta-analysis. Emerg Med J. 2020;37(4):232-9.

10. Gunes Ozaydin M, Guneysel O, Saridogan F, Ozaydin V. Are scoring systems sufficient for predicting mortality due to sepsis in the emergency department? Turk J Emerg Med. 2017;17(1):25-8.

11. Kim SJ, Hwang SO, Kim YW, Lee JH, Cha KC. Procalcitonin as a diagnostic marker for sepsis/septic shock in the emergency department; a study based on Sepsis-3 definition. Am J Emerg Med. 2019;37(2):272-6.

12. Subbe CP, Kruger M, Rutherford P, Gemmel L. Validation of a modified Early Warning Score in medical admissions. QJM. 2001;94(10):521-6.

13. Tavares RC, Vieira AS, Uchoa LV, Peixoto Júnior AA, Meneses FA. Validation of an early warning score in pre-intensive care unit. Rev Bras Ter Intensiva. 2008;20(2):124-7.

14. Kwon H, Kim YJ, Jo YH, Lee JH, Lee JH, Kim J, et al. The Korean Triage and Acuity Scale: associations with admission, disposition, mortality and length of stay in the emergency department. Int J Qual Health Care. 2019;31(6):449-55.

15. Park J, Lim T. Korean triage and acuity scale (KTAS). J Korean Soc Emerg Med. 2017;28(6):547-51.

16. Singer M, Deutschman CS, Seymour CW, Shankar-Hari M, Annane D, Bauer M, et al. The Third International Consensus Definitions for Sepsis and Septic Shock (Sepsis-3). JAMA. 2016;315(8):80110.

17. Charlson ME, Pompei P, Ales KL, MacKenzie CR. A new method of classifying prognostic comorbidity in longitudinal studies: development and validation. J Chronic Dis. 1987;40(5):373-83.

18. Christ M, Grossmann F, Winter D, Bingisser R, Platz E. Modern triage in the emergency department. Dtsch Arztebl Int. 2010;107(50):892-8. 
19. Lähdet EF, Suserud BO, Jonsson A, Lundberg L. Analysis of triage worldwide. Emerg Nurse. 2009;17(4):16-9.

20. Wang HE, Shapiro NI, Angus DC, Yealy DM. National estimates of severe sepsis in United States emergency departments. Crit Care Med. 2007;35(8):1928-36.

21. Yealy DM, Kellum JA, Huang DT, Barnato AE, Weissfeld LA, Pike F, et al. A randomized trial of protocol-based care for early septic shock. N Engl J Med. 2014;370(18):1683-93.

22. Fan SL, Miller NS, Lee J, Remick DG. Diagnosing sepsis - The role of laboratory medicine. Clin Chim Acta. 2016;460:203-10.

23. McLymont N, Glover GW. Scoring systems for the characterization of sepsis and associated outcomes. Ann Transl Med. 2016;4(24):527.

24. Jones AE, Shapiro NI, Roshon M. Implementing early goal-directed therapy in the emergency setting: the challenges and experiences of translating research innovations into clinical reality in academic and community settings. Acad Emerg Med. 2007;14(11):1072-8.

25. Carlbom DJ, Rubenfeld GD. Barriers to implementing protocol-based sepsis resuscitation in the emergency department--results of a national survey. Crit Care Med. 2007;35(11):2525-32.

26. Usman OA, Usman AA, Ward MA. Comparison of SIRS, qSOFA, and NEWS for the early identification of sepsis in the Emergency Department. Am J Emerg Med. 2019;37(8):1490-7.

27. Burch VC, Tarr G, Morroni C. Modified early warning score predicts the need for hospital admission and inhospital mortality. Emerg Med J. 2008;25(10):674-8.

\section{Tables}

Table 1. Baseline characteristics of study patients 


\begin{tabular}{|c|c|c|c|c|}
\hline Characteristics & $\begin{array}{l}\text { All patients } \\
(\mathrm{n}=5093)\end{array}$ & $\begin{array}{l}\text { Patients without } \\
\text { Septic shock } \\
(n=4698)\end{array}$ & $\begin{array}{l}\text { Patients with } \\
\text { septic shock } \\
(n=395)\end{array}$ & $p$ \\
\hline Age, year & $\begin{array}{l}63.0 \\
{[43.0 ; 77.0]}\end{array}$ & $61.0[42.0 ; 76.0]$ & $78.0[68.0 ; 83.0]$ & $<0.001$ \\
\hline Male patients, n (\%) & $2528(49.6)$ & $2330(49.6)$ & $198(50.1)$ & 0.839 \\
\hline \multicolumn{5}{|l|}{ Initial vital signs } \\
\hline SBP, mm Hg & $\begin{array}{l}124.0 \\
{[109.0 ; 140.0]}\end{array}$ & 125.0 [111.0;140.0] & $99.0[85.0 ; 127.0]$ & $<0.001$ \\
\hline Pulse rate, beats/min & $\begin{array}{l}94.0 \\
{[81.0 ; 108.0]}\end{array}$ & $94.0[81.0 ; 107.0]$ & $106.0[88.0 ; 122.0]$ & $<0.001$ \\
\hline $\begin{array}{l}\text { Respiratory rate, } \\
\text { breaths/min }\end{array}$ & $\begin{array}{l}20.0 \\
{[20.0 ; 22.0]}\end{array}$ & $20.0[20.0 ; 22.0]$ & $24.0[24.0 ; 28.0]$ & $<0.001$ \\
\hline Body temperature, ${ }^{\circ} \mathrm{C}$ & $\begin{array}{l}37.5 \\
{[36.8 ; 38.4]}\end{array}$ & $37.4[36.8 ; 38.4]$ & $37.8[36.7 ; 38.7]$ & 0.060 \\
\hline $\begin{array}{l}\text { Altered level of } \\
\text { consciousness, n (\%) }\end{array}$ & $566(11.1)$ & $280(6.0)$ & $286(72.4)$ & $<0.001$ \\
\hline \multicolumn{5}{|l|}{ Laboratory findings } \\
\hline WBC, $\times 10^{3}$ cells $/ \mathrm{mL}$ & $\begin{array}{l}11,017 \\
(7,179)\end{array}$ & $10,782(6,892)$ & $13,808(9,551)$ & $<0.001$ \\
\hline Albumin, $\mathrm{g} / \mathrm{dL}$ & $3.5(0.7)$ & $3.6(0.6)$ & $2.9(0.7)$ & $<0.001$ \\
\hline $\mathrm{BUN}, \mathrm{mg} / \mathrm{dL}$ & $17.4(12.8)$ & $16.5(11.6)$ & $28.4(19.7)$ & $<0.001$ \\
\hline Creatinine, $\mathrm{mg} / \mathrm{dL}$ & $1.14(1.08)$ & $1.10(1.06)$ & $1.48(1.28)$ & $<0.001$ \\
\hline CRP, mg/dL & $8.3(8.2)$ & $8.0(8.1)$ & $12.0(9.0)$ & $<0.001$ \\
\hline Glucose, mg/dL & $140.4(62.6)$ & $138.0(59.6)$ & $169.0(86.6)$ & $<0.001$ \\
\hline $\mathrm{CCl}$ & $3.0[0.0 ; 5.0]$ & $2.0[0.0 ; 5.0]$ & $5.0[4.0 ; 6.0]$ & $<0.001$ \\
\hline LTCF, n (\%) & $378(7.4)$ & $271(5.8)$ & $107(27.1)$ & $<0.001$ \\
\hline MEDS & $4.50(4.09)$ & $3.91(3.57)$ & $11.44(3.48)$ & $<0.001$ \\
\hline MEWS & $2.96(1.92)$ & $2.70(1.69)$ & $6.02(1.90)$ & $<0.001$ \\
\hline KTAS, n (\%) & & & & $<0.001$ \\
\hline 1 & $59(1.2 \%)$ & $28(0.6 \%)$ & 31 (7.8\%) & \\
\hline 2 & $507(10.0 \%)$ & $317(6.7 \%)$ & $190(48.3 \%)$ & \\
\hline 3 & $2755(54.1 \%)$ & 2596 (55.3\%) & $159(40.3 \%)$ & \\
\hline
\end{tabular}




\begin{tabular}{|lllll|}
\hline Characteristics & $\begin{array}{l}\text { All patients } \\
(\mathrm{n}=5093)\end{array}$ & $\begin{array}{l}\text { Patients without } \\
\text { Septic shock } \\
(\mathrm{n}=4698)\end{array}$ & $\begin{array}{l}\text { Patients with } \\
\text { septic shock } \\
(\mathrm{n}=395)\end{array}$ & $p$ \\
\hline 4 & $1645(32.3 \%)$ & $1632(34.7 \%)$ & $13(3.3 \%)$ & \\
\hline 5 & $127(2.5 \%)$ & $125(2.7 \%)$ & $2(0.5 \%)$ & $<0.001$ \\
\hline KTAS score & $2.75(0.71)$ & $2.68(0.67)$ & $3.59(0.70)$ & $<0.001$ \\
\hline KMEWS & $5.71(2.37)$ & $5.38(2.06)$ & $9.61(2.23)$ & $<0.001$ \\
\hline ICU admission, $\mathrm{n}(\%)$ & $250(4.9 \%)$ & $142(3.0 \%)$ & $108(27.3 \%)$ & $<0.001$ \\
\hline In-hospital mortality, $\mathrm{n}(\%)$ & $200(3.9 \%)$ & $127(2.7 \%)$ & $73(18.5 \%)$ & \\
\hline
\end{tabular}

Categorial variables are expressed by number (\%) and continuous variables are expressed by median [interquartile range] or mean (standard deviation).

Abbreviations: BUN, Blood urea nitrogen; $\mathrm{CCl}$, Charlson comorbidity index score; CRP, C-reactive protein; ICU, Intensive care unit; KTAS, Korean Triage Acuity Scale; LTCF, Long-term Care Facility; MEDS, Mortality in Emergency Department Sepsis; MEWS, Modified Early Warning Score; qSOFA, quick Sepsis-related Organ Failure Assessment; SBP, Systolic blood pressure; WBC, White blood cell

KTAS score; level 1: 5, level 2: 4, level 3: 3, level 4: 2, level 5: 1, KMEWS; Sum of the KTAS score and MEWS Table 2. Multivariable logistic regression analysis of factors associated with septic shock in patients in the emergency department

\begin{tabular}{|lllc|}
\hline Variables & OR & $95 \% \mathrm{Cl}$ & $p$ \\
\hline Age & 1.04 & $1.03-1.05$ & $<0.001$ \\
\hline Sex: male & 1.09 & $0.84-1.42$ & 0.509 \\
\hline Charlson Comorbidity Index & 1.01 & $0.95-1.09$ & 0.697 \\
\hline LTCF: Yes & 2.92 & $2.07-4.12$ & $<0.001$ \\
\hline KTAS score & 2.16 & $1.74-2.68$ & $<0.001$ \\
\hline MEWS & 2.09 & $1.93-2.27$ & $<0.001$ \\
\hline
\end{tabular}

Cl, confidence interval; KTAS, Korean Triage Acuity Scale; LTCF, Long-term Care Facility; MEWS, Modified Early Warning Score; OR, odds ratio;.

\section{Figures}




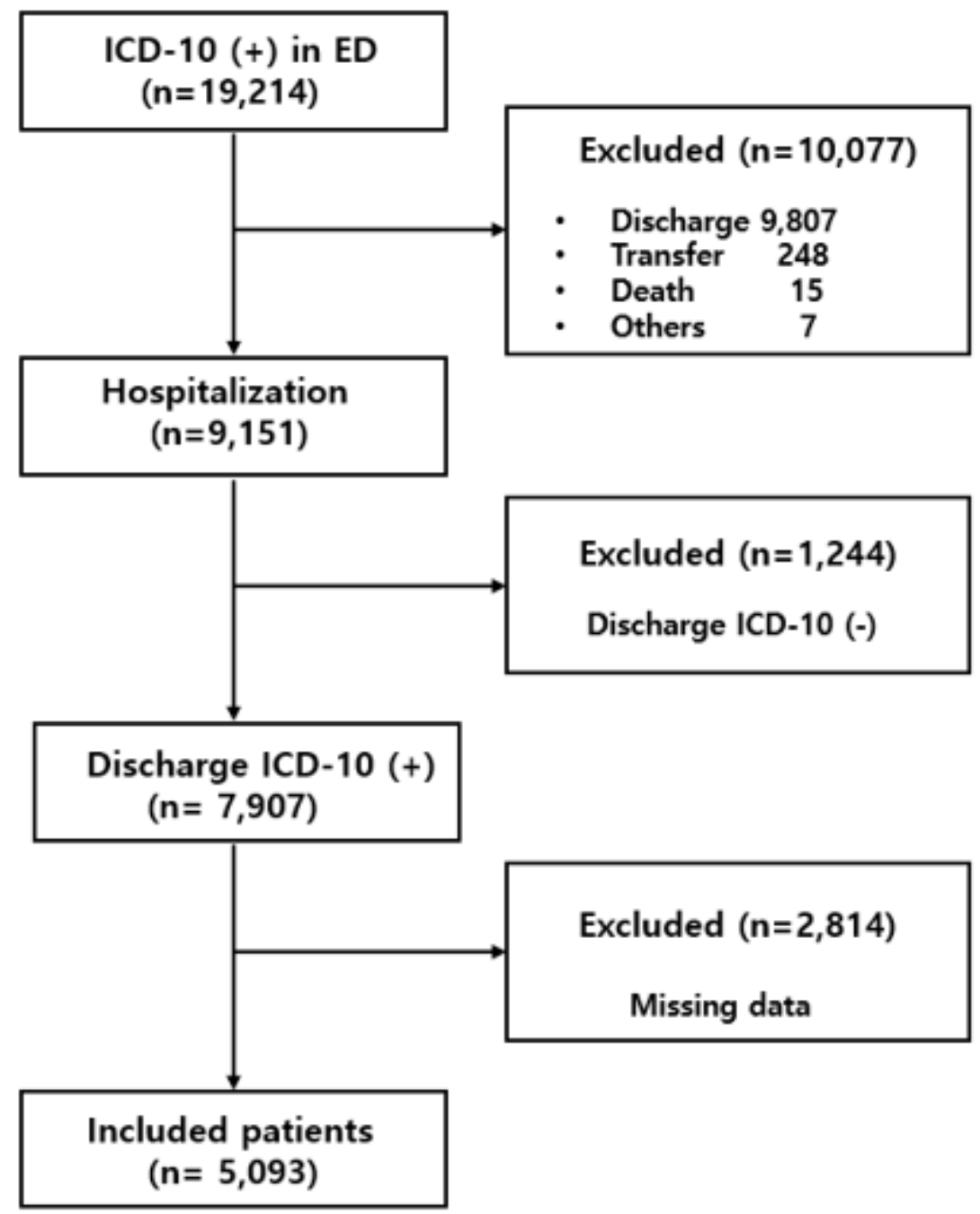

ICD-10=International Statistical Classification of Diseases and Related Health Problems $10^{\text {th }}$ Revision code. ICD-10 (+): Included ICD-10 codes were infection-related diagnoses.

Figure 1

Flow diagram for the study. 


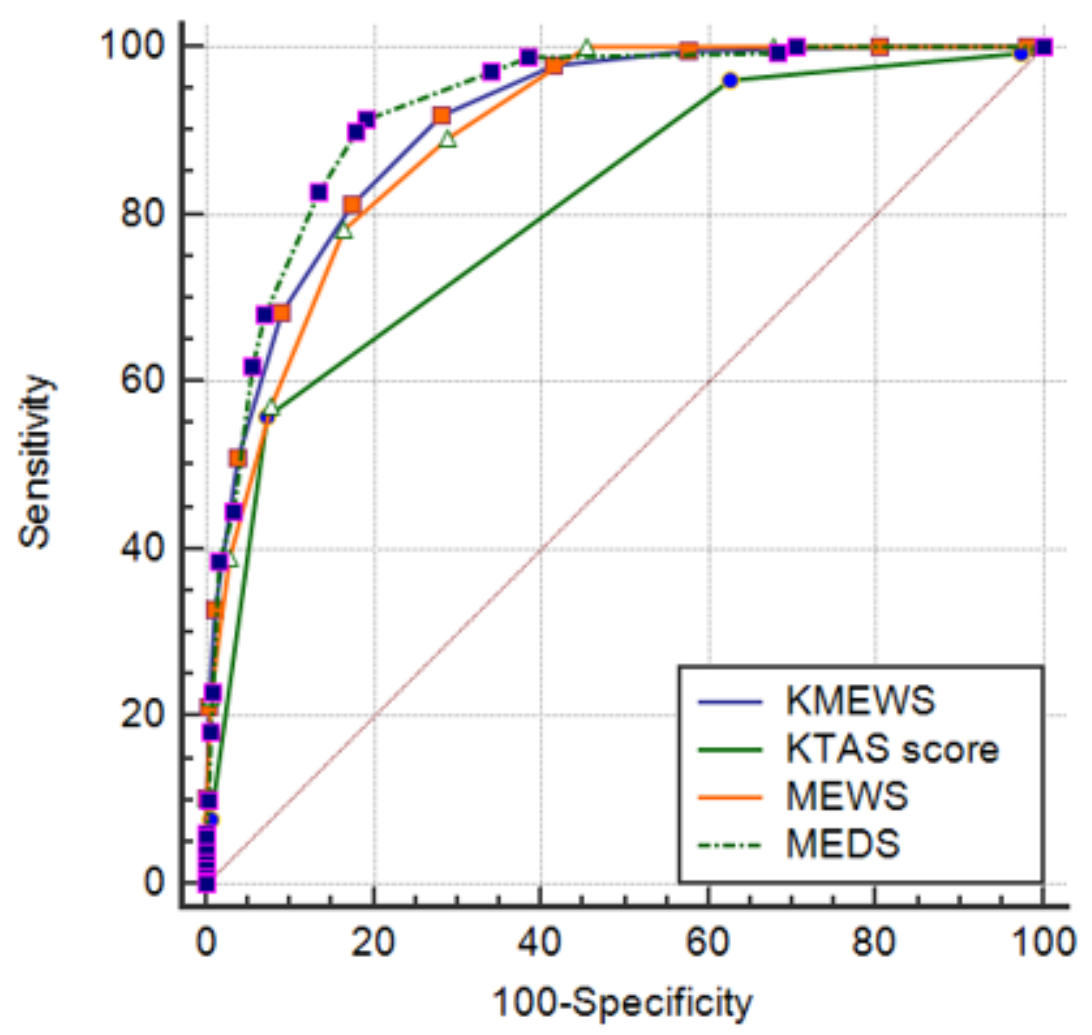

\begin{tabular}{lccc}
\hline Variables & AUC $(95 \% \mathrm{Cl})$ & $P$ & $\begin{array}{c}P \text { (equality compared } \\
\text { to KMEWS })\end{array}$ \\
\hline KMEWS & $0.910(0.902-0.918)$ & 0.006 & \\
MEWS & $0.896(0.887-0.904)$ & 0.006 & $<0.001$ \\
KTAS score & $0.809(0.798-0.819)$ & 0.010 & $<0.001$ \\
MEDS & $0.927(0.919-0.934)$ & 0.005 & $<0.019$
\end{tabular}

$\overline{\mathrm{AUC}}$, area under curve; $\mathrm{Cl}$, confidence interval; MEWS, Modified Early Warning Score; KTAS, Korean Triage Acuity Scale; MEDS, Mortality in Emergency Department Sepsis; KMEWS, Sum of the KTAS score and MEWS

The AUCs of the models were calculated and tested mutually for significance by DeLong equality tests.

\section{Figure 2}

Analysis of receiver-operating characteristics curve for predicting septic shock The AUCs of the models were calculated and tested mutually for significance by DeLong equality tests. AUC, area under curve; $\mathrm{Cl}$, confidence interval; KMEWS, Sum of the KTAS score and MEWS; KTAS, Korean Triage Acuity Scale; MEDS, Mortality in Emergency Department Sepsis; MEWS, Modified Early Warning Score. 


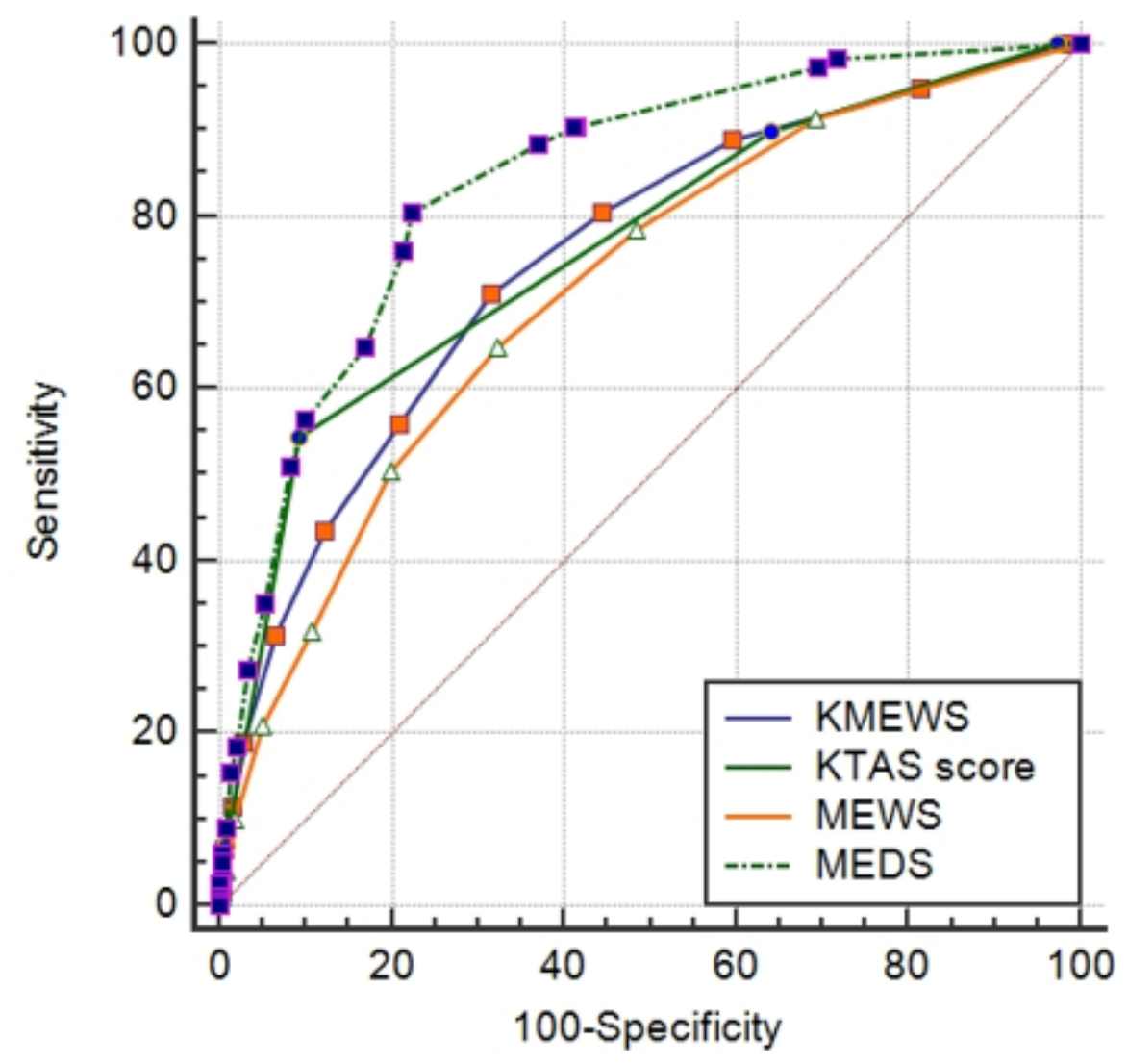

\begin{tabular}{lccc}
\hline Variables & AUC $(95 \% \mathrm{Cl})$ & $p$ & $\begin{array}{c}p \text { (equality compared } \\
\text { to KMEWS })\end{array}$ \\
\hline KMEWS & $0.752(0.740-0.764)$ & 0.018 & \\
MEWS & $0.717(0.704-0.729)$ & 0.018 & $<0.001$ \\
KTAS score & $0.764(0.752-0.776)$ & 0.017 & 0.301 \\
MEDS & $0.844(0.834-0.854)$ & 0.013 & $<0.001$
\end{tabular}

$\overline{\mathrm{AUC}}$, area under curve; $\mathrm{Cl}$, confidence interval; MEWS, Modified Early Warning Score; KTAS, Korean Triage Acuity Scale; MEDS, Mortality in Emergency Department Sepsis; KMEWS, Sum of the KTAS score and MEWS

The AUCs of the models were calculated and tested mutually for significance by DeLong equality tests.

\section{Figure 3}

Analysis of receiver-operating characteristics curve for predicting mortality The AUCs of the models were calculated and tested mutually for significance by DeLong equality tests. AUC, area under curve; $\mathrm{Cl}$, confidence interval; KMEWS, Sum of the KTAS score and MEWS; KTAS, Korean Triage Acuity Scale; MEDS, Mortality in Emergency Department Sepsis; MEWS, Modified Early Warning Score. 


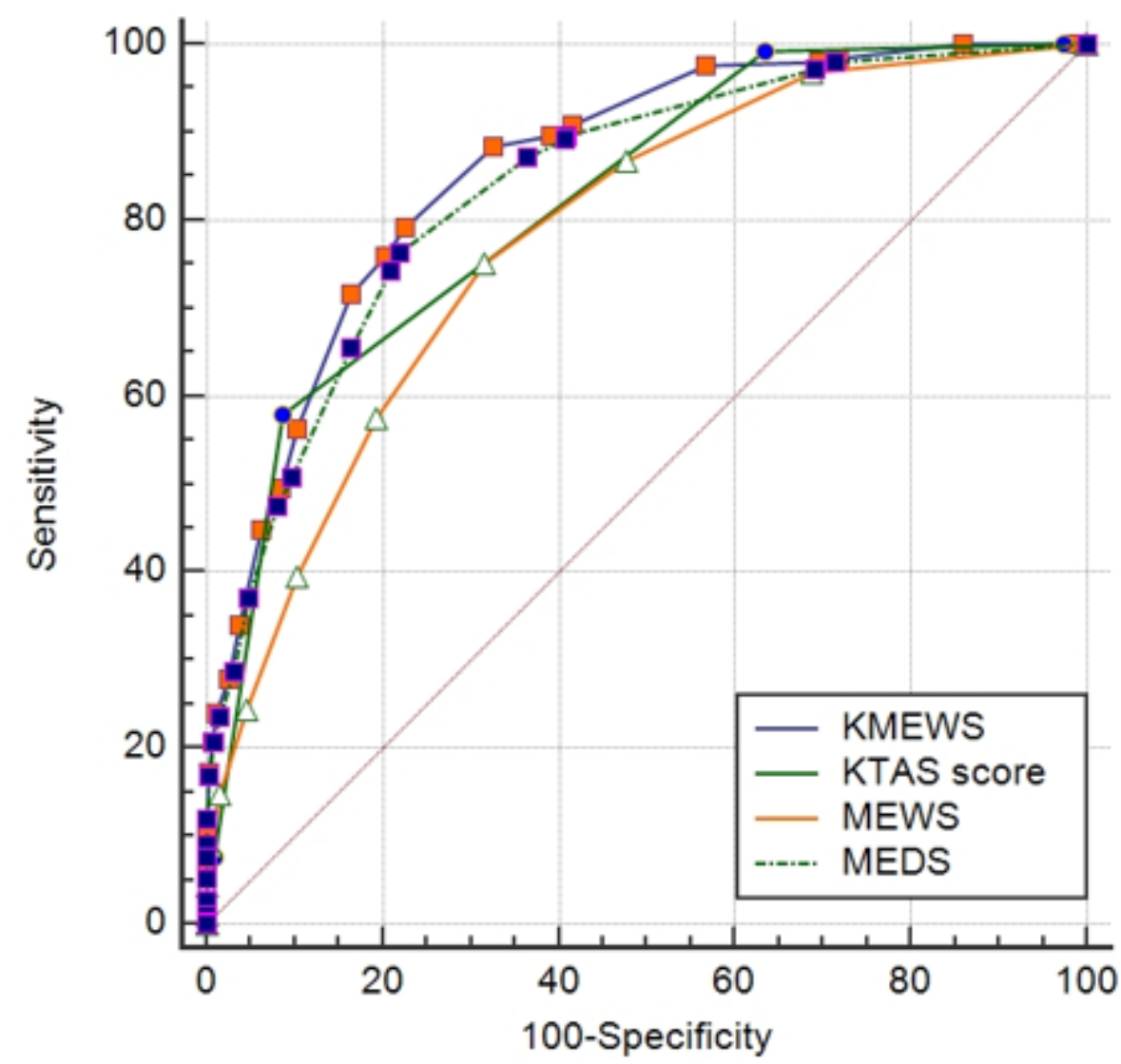

\begin{tabular}{lccc}
\hline Variables & AUC $(95 \% \mathrm{Cl})$ & $p$ & $\begin{array}{r}p \text { (equality compared } \\
\text { to KMEWS })\end{array}$ \\
\hline KMEWS & $0.826(0.816-0.837)$ & 0.011 & \\
MEWS & $0.782(0.770-0.793)$ & 0.014 & $<0.001$ \\
KTAS score & $0.821(0.810-0.831)$ & 0.011 & 0.598 \\
MEDS & $0.839(0.829-0.849)$ & 0.012 & 0.269
\end{tabular}

$\overline{\mathrm{AUC}}$, area under curve; $\mathrm{Cl}$, confidence interval; MEWS, Modified Early Warning Score; KTAS, Korean Triage Acuity Scale; MEDS, Mortality in Emergency Department Sepsis; KMEWS, Sum of the KTAS score and MEWS

The AUCs of the models were calculated and tested mutually for significance by DeLong equality tests.

Figure 4

Analysis of receiver-operating characteristics curve for predicting ICU admission The AUCs of the models were calculated and tested mutually for significance by DeLong equality tests. AUC, area under curve; $\mathrm{Cl}$, confidence interval; KMEWS, Sum of the KTAS score and MEWS; KTAS, Korean Triage Acuity Scale; MEDS, Mortality in Emergency Department Sepsis; MEWS, Modified Early Warning Score.

\section{Supplementary Files}


This is a list of supplementary files associated with this preprint. Click to download.

- supplementaryTable1.docx

- supplementaryTable2.docx 